\title{
Operations on Signed Distance Functions ${ }^{a}$
}

\author{
Csaba Bálint, ${ }^{b}$ Gábor Valasek ${ }^{b}$ and Lajos Gergó ${ }^{b}$
}

\begin{abstract}
We present a theoretical overview of signed distance functions and analyze how this representation changes when applying an offset transformation. First, we analyze the properties of signed distance and the sets they describe.

Second, we introduce our main theorem regarding the distance to an offset set in $(X,\|\cdot\|)$ strictly normed Banach spaces. An offset set of $D \subseteq X$ is the set of points equidistant to $D$. We show when such a set can be represented by $f(\boldsymbol{x})-c=0$, where $c \neq 0$ denotes the radius of the offset. Finally, we apply these results to gain a deeper insight into offsetting surfaces defined by signed distance functions.
\end{abstract}

Keywords: signed distance functions, sphere tracing, computer graphics

\section{Introduction}

Surface representations for real-time graphics rely on linear approximations. With the advent of hardware accelerated tessellation units, parametric surfaces gained momentum in real-time computer graphics; however, implicit mappings are still considered infeasible for high-performance applications $[2,4,6,7,9,13]$.

Nevertheless, implicit functions simplify some otherwise challenging operations. For example, blending between different shapes does not necessitate the explicit representation of the target topologies when both objects are represented implicitly $[3,19]$. Similarly, the result of set operations on these objects can be trivially computed $[7,12,14,15]$.

Our paper focuses on a particular class of implicit representations, signed distance functions (SDFs). Hart noted in [10] that SDFs could be rendered efficiently using a technique called sphere tracing $[2,9,16]$. This algorithm and the constant evolution of GPUs opened up the possibility of incorporating implicit representations into real-time applications, as exemplified by $[1,6,18]$ more recently.

We discuss this class of functions and highlight their theoretical aspects that have practical consequences in rendering. In particular, we focus on offsetting SDF representations. Although both offsets and SDFs are simple concepts, their

\footnotetext{
${ }^{a}$ The project has been supported by the European Union, co-financed by the European Social Fund (EFOP-3.6.3-VEKOP-16-2017-00001).

${ }^{b}$ Eötvös Loránd University, E-mail: \{csabix, valasek, gergo\}@inf .elte.hu
} 


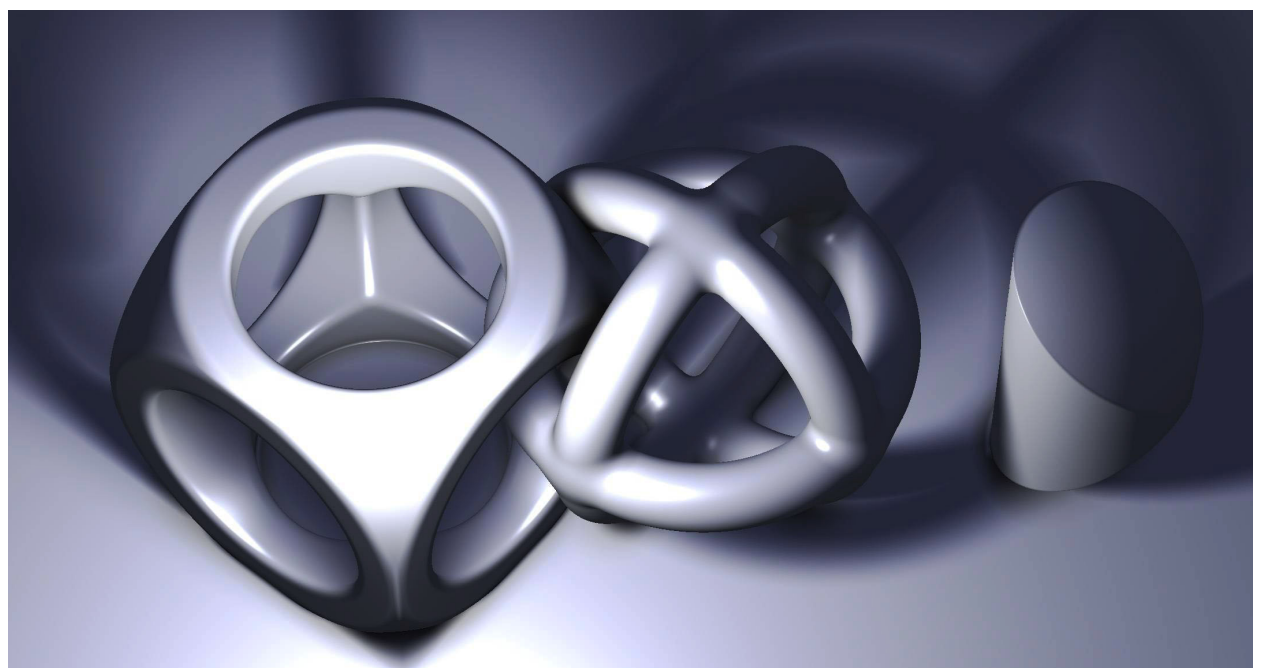

Figure 1: A scene modeled with and rendered using signed distance functions.

combination does not always yield the expected simplicity when one tries to find a representation for the result, as highlighted in Section 6. Our paper begins with a set-theoretic overview in Section 2. We base our theorems upon these results.

Section 3 present a general algorithm for displaying surfaces defined by implicit functions, whereas Section 4 demonstrates the power SDFs provide in speeding up such tasks and their practical importance.

In Section 5, we propose a slightly different definition for signed distance function than seen in [10]. We show that the two definitions are equivalent.

We present our main result in Section 6 . We show that it is possible to represent the radius $c \neq 0$ offset of $f(\boldsymbol{x})=0$ by $f(\boldsymbol{x})-c=0$; however, $f-c$ only produces a signed distance function on the subset of $\mathbb{R}^{3}$ for which $\frac{f(\boldsymbol{x})}{c} \geq 1$.

It has been observed that adding a constant value to a signed distance function produces a function that defines the offset set of the original surface $[8,10,17]$. In this paper, we analyze this operation mathematically and explain the reasons behind the effectiveness and limitations of the practical solutions.

\section{Set-theoretic basics}

This section reviews the definitions and results from the literature our paper relies on. Dyer et al. explain the topic in more detail in [5]. Let $(X, d)$ denote a metric space. We also use $d: X \times X \rightarrow[0,+\infty]$ to denote the distance to a set.

Definition 1 (Distance to set). Let $A \subseteq X, \boldsymbol{p} \in X$. Then

$$
d(\boldsymbol{p}, A):=\inf _{\boldsymbol{a} \in A} d(\boldsymbol{p}, \boldsymbol{a})
$$


denotes the distance of $\boldsymbol{p}$ from the set $A$. Let $\inf \emptyset:=+\infty$.

Definition 2 (Neighborhood). Let us denote the $r>0$ radius neighborhood of an element $\boldsymbol{p} \in X$ by

$$
\mathcal{S}_{r}(\boldsymbol{p}):=\{\boldsymbol{x} \in X: d(\boldsymbol{x}, \boldsymbol{p})<r\} .
$$

$A \subseteq X$ is open if $\forall \boldsymbol{a} \in A, \exists \epsilon>0: \mathcal{S}_{\epsilon}(\boldsymbol{a}) \subseteq A$. The set $B \subseteq X$ is closed if $X \backslash B$ is open. Note that $\emptyset$ and $X$ are both closed and open.

$C \subseteq X$ is compact if every open covering of it can be reduced to be of finite cardinality. A compact set is closed and bounded, i.e. $\exists R>0$ such that $C \subseteq \mathcal{S}_{R}(\mathbf{0})$. A bounded and closed set is compact if $X$ is a finite dimensional metric space, for example $X=\mathbb{R}^{3}$.

Lemma 1 (Existence of extremal element). Suppose $A \subseteq X$ is closed and $\boldsymbol{x} \in X$ where $(X, d)$ is a complete metric space. Then

$$
\exists \boldsymbol{a} \in A: d(\boldsymbol{x}, A)=d(\boldsymbol{x}, \boldsymbol{a})
$$

The proof for Lemma 1 can be found in [11] on page 102 for $\mathbb{R}^{n}$, the proof is analogous for this case $[11,5]$.

Furthermore, we denote the interior of the set $A \subseteq X$ as

$$
\operatorname{int} A:=\left\{\boldsymbol{a} \in A \mid \exists \epsilon>0: \mathcal{S}_{\epsilon}(\boldsymbol{a}) \subseteq A\right\}
$$

The closure of $A \subseteq X$ is

$$
\bar{A}:=\left\{\boldsymbol{a} \in X \mid \forall \epsilon>0: \mathcal{S}_{\epsilon}(\boldsymbol{a}) \cap A \neq \emptyset\right\}
$$

The boundary of $A$ is denoted by $\partial A:=\bar{A} \backslash \operatorname{int} A$. For any set $A \subseteq X$ it follows from the definitions that int $A$ is open, $\bar{A}$ and $\partial A$ are closed sets.

\section{Raymarching}

From now on, let us consider surfaces defined by an $f: \mathbb{R}^{3} \rightarrow \mathbb{R}$ implicit function, such that the surface is the $\{f \equiv 0\}:=\left\{\boldsymbol{x} \in \mathbb{R}^{3} \mid f(\boldsymbol{x})=0\right\}$ level-set. For example, the characteristic function $1-\mathcal{X}_{D}=\mathcal{X}_{\mathbb{R}^{3} \backslash D}: \mathbb{R}^{3} \rightarrow\{0,1\}$ is an implicit function of any $D \subseteq \mathbb{R}^{3}$ set.

A ray is a half line originating from a particular point, for example, the camera. Let us represent rays by their origin $\boldsymbol{p} \in \mathbb{R}^{3}$ and unit length direction vector $\boldsymbol{v} \in \mathbb{R}^{3},\|\boldsymbol{v}\|_{2}=1$. Then a ray is written as

$$
\boldsymbol{s}(t):=\boldsymbol{s}_{\boldsymbol{p}, \boldsymbol{v}}(t):=\boldsymbol{p}+t \cdot \boldsymbol{v} \in \mathbb{R}^{3} \quad(t \geq 0) .
$$

Therefore, the ray-surface intersection problem can be expressed as a root finding problem. We need to find the smallest positive root of the

$$
f \circ s:[0,+\infty) \rightarrow \mathbb{R}
$$




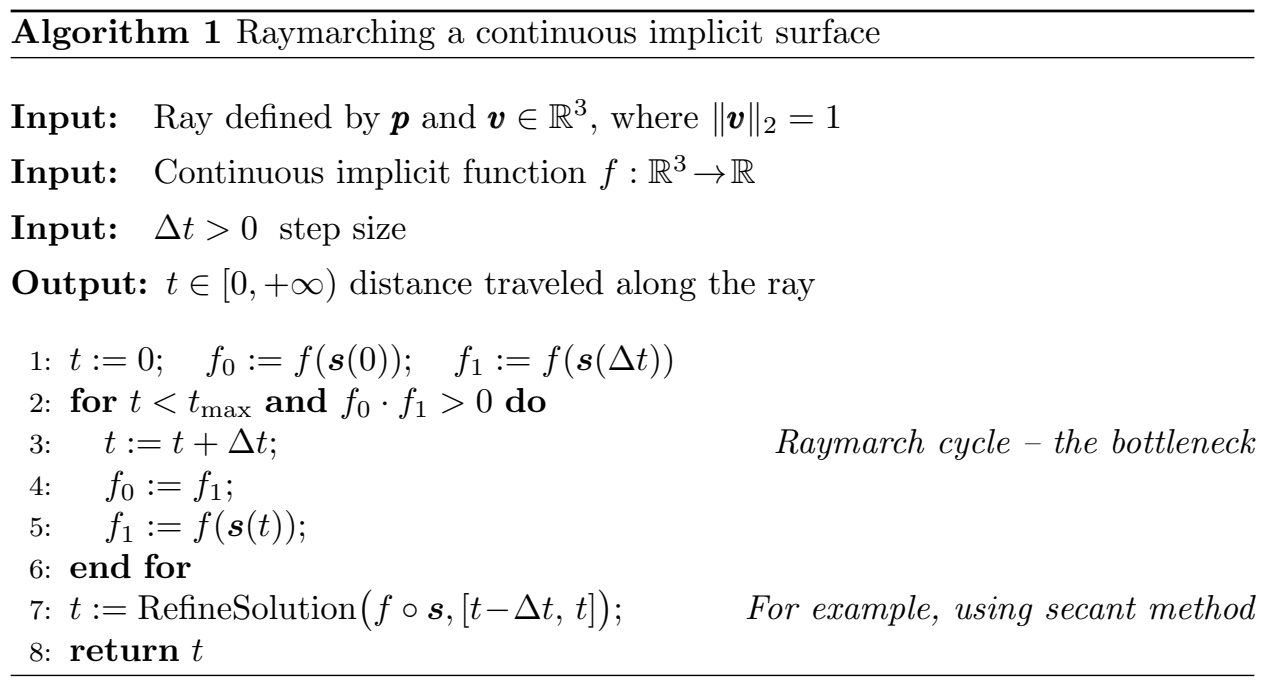

composite function. Usually, one can infer that $f$ is continuous in which case raymarching that is shown in Algorithm 1 can be used to find an approximate solution. The method takes $\Delta t$ sized steps along the ray looking for two consecutive values of different signs.

Despite being a popular algorithm for implicit surface rendering, raymarching is expensive, and it may even skip over solutions, causing visible artifacts. To provide a better ray tracing algorithm, $f$ needs to be restricted even further which is explained in the next section.

\section{Sphere Tracing}

Throughout this section, we adapt the definitions from Hart [10]. Let us consider the Banach-space $\left(\mathbb{R}^{3},\|\cdot\|_{2}\right)$ where we denote the induced metric as $d(\boldsymbol{x}, \boldsymbol{y}):=$ $\|\boldsymbol{y}-\boldsymbol{x}\|_{2}\left(\boldsymbol{x}, \boldsymbol{y} \in \mathbb{R}^{3}\right)$.

Definition 3 (Distance function). $f: \mathbb{R}^{3} \rightarrow[0,+\infty)$ is a distance function if

$$
f(\boldsymbol{p})=d(\boldsymbol{p},\{f \equiv 0\}) \quad\left(\forall \boldsymbol{p} \in \mathbb{R}^{3}\right) .
$$

Example. The distance function of the unit sphere is

$$
f_{\text {sphere }}(\boldsymbol{p})=d\left(\boldsymbol{p}, \mathcal{S}_{1}(\mathbf{0})\right)=\max \left(\|\boldsymbol{p}\|_{2}-1,0\right)\left(\boldsymbol{p} \in \mathbb{R}^{3}\right) .
$$

Definition 4 (Unbounding sphere). The unbounding sphere for the distance function $f: \mathbb{R}^{3} \rightarrow[0,+\infty)$ at $\boldsymbol{p} \in \mathbb{R}^{3}$ is the open neighbourhood $S_{f(\boldsymbol{p})}(\boldsymbol{p})$.

It follows from Definition 3 that there are no surface points closer to $\boldsymbol{p}$ than $f(\boldsymbol{p})$, i.e. $\mathcal{S}_{f(\boldsymbol{p})}(\boldsymbol{p}) \cap\{f \equiv 0\}=\emptyset$. 


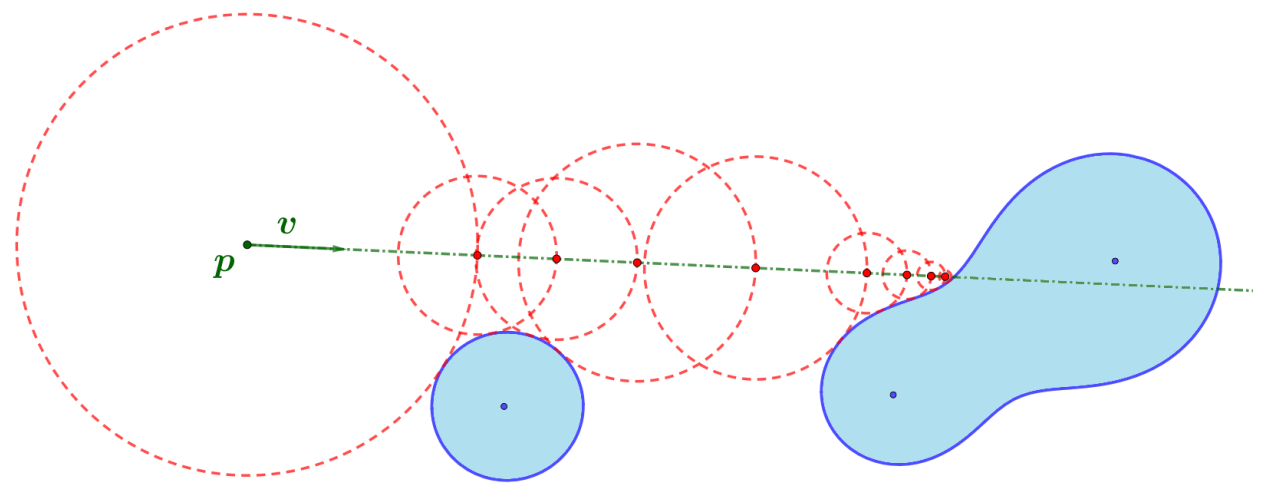

Figure 2: The sphere tracing algorithm takes distance sized steps, thereby it does not overstep a solution, yet it converges quickly. Each step defines an unbounding sphere that is disjoint from the surface.

Algorithm 2 Sphere tracing a surface defined by a distance function

Input: $\quad$ Ray defined by $\boldsymbol{p}$ and $\boldsymbol{v} \in \mathbb{R}^{3}$, where $\|\boldsymbol{v}\|_{2}=1$

Input: Distance function $f: \mathbb{R}^{3} \rightarrow \mathbb{R}$

Output: $t \in[0,+\infty)$ distance traveled along the ray

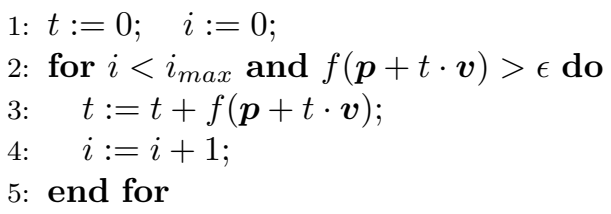

This property shows that sphere tracing shown in Algorithm 2 can be used to find the first ray-surface intersection robustly. The algorithm iteratively takes distance-sized steps along the ray; thus no ray-surface intersection is skipped while large empty spaces are traversed quickly.

As a consequence of the above, as we approach the surface along the ray, the distance to the surface cannot change more than what we have travelled. We generalize this using the Lemma 2 and Corollary 1 below.

Lemma 2. Let the set $A \subseteq \mathbb{R}^{n}$ be a closed set and $\boldsymbol{x}, \boldsymbol{y} \in \mathbb{R}^{n}$. Then

$$
|d(\boldsymbol{x}, A)-d(\boldsymbol{y}, A)| \leq d(\boldsymbol{x}, \boldsymbol{y})
$$

Proof. Since $A$ is a closed set, there exist $\boldsymbol{x}^{\prime}, \boldsymbol{y}^{\prime} \in A$ such that $d\left(\boldsymbol{x}, \boldsymbol{x}^{\prime}\right)=d(\boldsymbol{x}, A)$ and $d\left(\boldsymbol{y}, \boldsymbol{y}^{\prime}\right)=d(\boldsymbol{y}, A)$ according to Lemma 1. Using the definition of the distance, we provide a lower bound to $d\left(\boldsymbol{x}, \boldsymbol{y}^{\prime}\right)$ and $d\left(\boldsymbol{y}, \boldsymbol{x}^{\prime}\right)$ respectively. The upper bound 
is given by the triangle inequality in the $\boldsymbol{x} \boldsymbol{y} \boldsymbol{y}^{\prime}$ and $\boldsymbol{y} \boldsymbol{x} \boldsymbol{x}^{\prime}$ triangles, respectively:

$$
\begin{aligned}
& d\left(\boldsymbol{x}, \boldsymbol{x}^{\prime}\right) \leq d\left(\boldsymbol{x}, \boldsymbol{y}^{\prime}\right) \leq d(\boldsymbol{x}, \boldsymbol{y})+d\left(\boldsymbol{y}, \boldsymbol{y}^{\prime}\right), \\
& d\left(\boldsymbol{y}, \boldsymbol{y}^{\prime}\right) \leq d\left(\boldsymbol{y}, \boldsymbol{x}^{\prime}\right) \leq d(\boldsymbol{x}, \boldsymbol{y})+d\left(\boldsymbol{x}, \boldsymbol{x}^{\prime}\right) .
\end{aligned}
$$

Using (1) for the upper bound and (2) for the lower bound of $d\left(\boldsymbol{x}, \boldsymbol{x}^{\prime}\right)$ we have:

$$
d\left(\boldsymbol{y}, \boldsymbol{y}^{\prime}\right)-d(\boldsymbol{x}, \boldsymbol{y}) \leq d\left(\boldsymbol{x}, \boldsymbol{x}^{\prime}\right) \leq d\left(\boldsymbol{y}, \boldsymbol{y}^{\prime}\right)+d(\boldsymbol{x}, \boldsymbol{y})
$$

This proves Lemma 2 .

Definition 5 (Lipschitz constant). Let the function $f: \mathbb{R}^{3} \rightarrow \mathbb{R}$ be arbitrary, we define the set of Lipschitz constants as

$$
\operatorname{Lip} f:=\left\{L>0: \forall \boldsymbol{x}, \boldsymbol{y} \in \mathbb{R}^{3}:|f(\boldsymbol{x})-f(\boldsymbol{y})| \leq L \cdot d(\boldsymbol{x}, \boldsymbol{y})\right\} .
$$

The function $f$ is Lipschitz continuous if $\operatorname{Lip} f \neq \emptyset$.

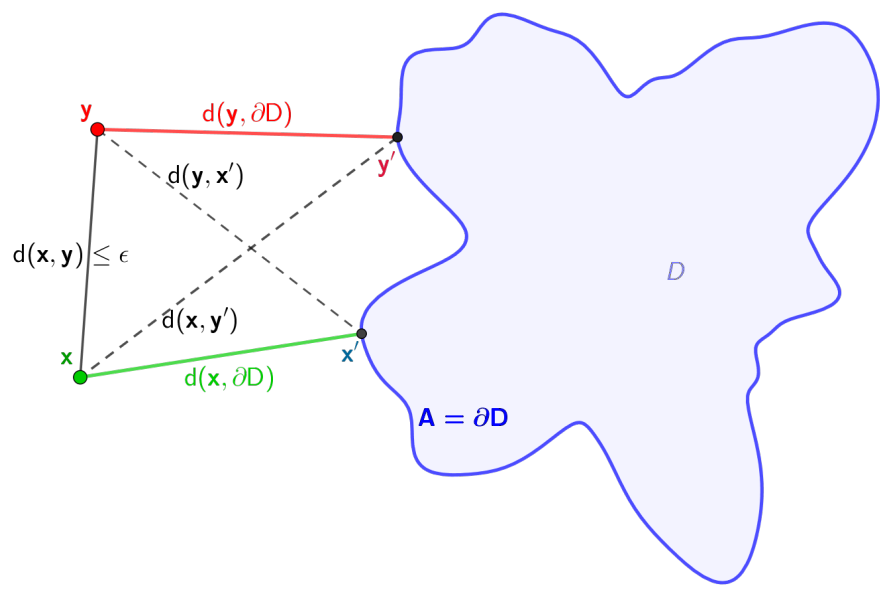

Figure 3: A visualization for the proof of Lemma 2 and Proposition 1.

Corollary 1. Every signed distance function is Lipschitz continuous and their smallest Lipschitz constant is 1. Formally:

$$
\forall f: \mathbb{R}^{3} \rightarrow \mathbb{R} S D F \quad: \inf \operatorname{Lip} f=\min \operatorname{Lip} f=1 \text {. }
$$

Proof. First, the Lemma 2 above implies that Lip $f \geq 1$ element-wise with $D:=A$. Second $1 \in \operatorname{Lip} f$, because if $\boldsymbol{y}:=\boldsymbol{x}^{\prime}$, then $\boldsymbol{y}=\boldsymbol{x}^{\prime}=\boldsymbol{y}^{\prime} \in A$ in the proof, then inequalities turn to equities in Equation 1. 


\section{Signed Distance Functions}

Definition $6(\mathrm{SDF})$. If $f: \mathbb{R}^{3} \rightarrow \mathbb{R}$ is continuous and $|f|$ is a distance function, then $f$ is a signed distance function.

Signed distance functions (SDFs) can represent an entire volume by classifying the points of $\mathbb{R}^{3}$ belonging to its 'interior' $(\{f<0\})$, 'exterior' $(\{f>0\})$, or to the surface $(\{f \equiv 0\})$. For example, $\mathbb{R}^{3} \ni \boldsymbol{p} \rightarrow\|\boldsymbol{p}\|_{2}-1 \in[-1,+\infty)$ is a signed distance function of the unit sphere.

Note that distance functions are a subset of SDFs, but they cannot differentiate between interior and surface points. For signed distance functions, we give the following equivalent definition:

Proposition 1 (SDF equivalence). The function $f: \mathbb{R}^{3} \rightarrow \mathbb{R}$ is a signed distance function if, and only if there exists $a \emptyset \neq D \subseteq \mathbb{R}^{3}$ set for which

$$
f(\boldsymbol{p})=\left\{\begin{array}{rl}
d(\boldsymbol{p}, \partial D) & \text { if } \boldsymbol{p} \notin D \\
-d(\boldsymbol{p}, \partial D) & \text { if } \boldsymbol{p} \in D
\end{array} .\right.
$$

Proof. First, let us assume that $f$ is defined according to equation (4). In this case, it follows that $|f|$ is a distance function of the $\partial D=\{f \equiv 0\}$ set. Using Lemma 2 with $A:=\partial D$, with $\boldsymbol{x}, \boldsymbol{y} \in\{f \geq 0\} \subseteq \mathbb{R}^{3}$ we know that

$$
|f(\boldsymbol{x})-f(\boldsymbol{y})|=|d(\boldsymbol{x}, \partial D)-d(\boldsymbol{y}, \partial D)| \leq d(\boldsymbol{x}, \boldsymbol{y}),
$$

and therefore, $f$ is uniformly continuous function on the set $\{f \geq 0\}$. One can analogously show that $f$ is continuous on the set $\{f \leq 0\}$.

Assuming that $|f|$ is a distance function where $f: \mathbb{R}^{3} \rightarrow \mathbb{R}$ is a continuous function, we have to show that the $D:=\{f \leq 0\}$ set satisfy equation (4). It indeed does, because $\partial D=\{f \equiv 0\}$, and $|f(\boldsymbol{p})|=d(\boldsymbol{p},\{f \equiv 0\})$, and if, for example, $f(\boldsymbol{p})>0$, then $f(\boldsymbol{p})=d(\boldsymbol{p}, \partial D)$ and $\boldsymbol{p} \notin D$.

Hart [10] defined signed distance functions that are distance functions in absolute value. Definition 1 is similar to that of Hart, but the represented object $D$ appears in it. Moreover, the sign is not allowed to jump on the same side of the surface, so there is a distinct "inside" and "outside" region associated with the surface. However, this intuitive definition lacks the simplicity of the original, hence the need for Definition 6.

\section{Offset theorem}

Let us investigate the geometric operation of offsetting on SDF representations.

Definition 7 (Offset surface). The offset surface at signed distance $c \in \mathbb{R}$ of the surface defined by the $S D F f: \mathbb{R}^{3} \rightarrow \mathbb{R}$ is the $\{f \equiv c\}$ (level-)set. 
Intuitively, offsets are obtained by inflating or deflating an initial volume by some fixed radius $c \in \mathbb{R}$. Contrary to the naive assumption, however, offsets cannot be represented by $f(\boldsymbol{x})-c=0$ in general, see the counterexample on Figure 5 . Nevertheless, there's a subset of $\mathbb{R}^{3}$ where the SDF of the offset can be written this way, as shown in Theorem 1.

First, we define strict convexity. Strictly convex Banach spaces include $\mathbb{R}^{n}, \mathbb{C}^{n}$, and $\mathrm{L}^{p}$ spaces with $p$-norms, if $1<p<+\infty$.

Definition 8 (Strictly convex normal space). The $(X,\|\cdot\|)$ normal space is strictly convex, if for all $\boldsymbol{x}, \boldsymbol{y}, \boldsymbol{z} \in X$, the following holds:

$$
d(\boldsymbol{x}, \boldsymbol{z})+d(\boldsymbol{z}, \boldsymbol{y})=d(\boldsymbol{x}, \boldsymbol{y}) \Longleftrightarrow \exists \lambda \in[0,1]: \boldsymbol{z}=(1-\lambda) \cdot \boldsymbol{x}+\lambda \cdot \boldsymbol{y},
$$

where $d(\boldsymbol{x}, \boldsymbol{y})$ denotes the induced metric, i.e. $d(\boldsymbol{x}, \boldsymbol{y}):=\|\boldsymbol{y}-\boldsymbol{x}\|(\boldsymbol{x}, \boldsymbol{y} \in X)$.

Second, the definition of the open offset set follows, which is a generalization of neighborhood in Definition 2.

Definition 9 (Offset set). For any $D \subseteq X$ in the metric space $(X, d)$, one can define an open offset set from $D$ with $r \geq 0$ range, as

$$
\mathcal{S}_{r}(D):=\{\boldsymbol{x} \in X: d(\boldsymbol{x}, D)<r\} .
$$

Finally, we present the main contribution of this paper in the following

Theorem 1 (Offset theorem). Let $(X,\|\cdot\|)$ be a strictly convex Banach space and $D \subseteq X$ closed. Then for any $c \geq 0$,

$$
\forall \boldsymbol{p} \in X \backslash \mathcal{S}_{c}(D) \quad: \quad d(\boldsymbol{p}, D)-c=d\left(\boldsymbol{p}, \mathcal{S}_{c}(D)\right)
$$

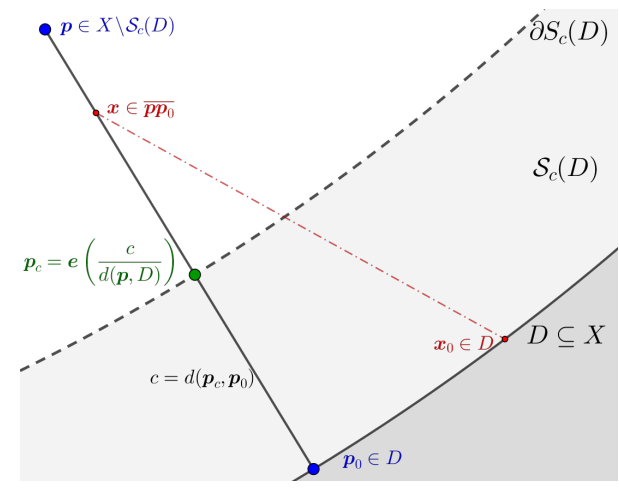

(a) Proof of $d(\boldsymbol{p}, D)-c \geq d\left(\boldsymbol{p}, \mathcal{S}_{c}(D)\right)$

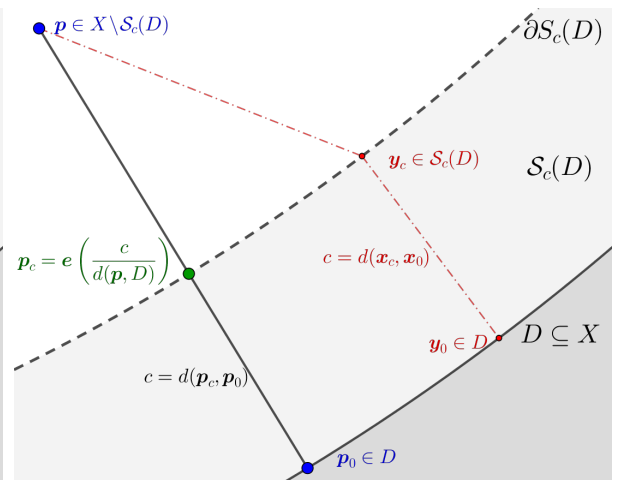

(b) Proof of $d(\boldsymbol{p}, D)-c \leq d\left(\boldsymbol{p}, \mathcal{S}_{c}(D)\right)$

Figure 4: A visualization of the proof for the offset theorem. 
Proof. Since the set containing the single element $\{\boldsymbol{p}\} \subset \mathbb{R}^{3}$ is compact and $D$ is closed, the extremal points exist between the two sets according to Lemma 1:

$$
\exists \boldsymbol{p}_{0} \in D: d(\boldsymbol{p}, D)=d\left(\boldsymbol{p}, \boldsymbol{p}_{0}\right) .
$$

The $\boldsymbol{e}(t):=(1-t) \cdot \boldsymbol{p}_{0}+t \cdot \boldsymbol{p} \in X,(t \in[0,1])$ is the parametric form of the $\overline{\boldsymbol{p}_{0} \boldsymbol{p}}$ line segment. First we show that

$$
\forall \boldsymbol{x} \in \overline{\boldsymbol{p}_{0} \boldsymbol{p}}: d\left(\boldsymbol{x}, \boldsymbol{p}_{0}\right)=d(\boldsymbol{x}, D) .
$$

Let us prove this by contradiction: let $\boldsymbol{x}_{0} \in D$ such that $d\left(\boldsymbol{x}, \boldsymbol{x}_{0}\right)<d\left(\boldsymbol{x}, \boldsymbol{p}_{0}\right)$. Using the definition of distance to the set, the triangle inequality in $\boldsymbol{x}_{0} \boldsymbol{p}_{0}$, the indirect assumption, and the strict concavity, in order, we have the following:

$$
\begin{aligned}
d(\boldsymbol{p}, D) & \leq d\left(\boldsymbol{p}, \boldsymbol{x}_{0}\right) \leq d(\boldsymbol{p}, \boldsymbol{x})+d\left(\boldsymbol{x}, \boldsymbol{x}_{0}\right) \\
& <d(\boldsymbol{p}, \boldsymbol{x})+d\left(\boldsymbol{x}, \boldsymbol{p}_{0}\right)=d\left(\boldsymbol{p}, \boldsymbol{p}_{0}\right)=d(\boldsymbol{p}, D)
\end{aligned}
$$

Which is a contradiction, so all $\boldsymbol{x} \in \overline{\boldsymbol{p}_{0} \boldsymbol{p}}$, the $\boldsymbol{p}_{0}$ is a closest point in $D$. When $\boldsymbol{x}=\boldsymbol{e}(t)$, one can deduce that the distance from $D$ along $\boldsymbol{e}$ is linear:

$$
d(\boldsymbol{e}(t), D)=d\left(\boldsymbol{e}(t), \boldsymbol{p}_{0}\right)=t \cdot d\left(\boldsymbol{p}, \boldsymbol{p}_{0}\right) \quad(t \in[0,1]) .
$$

Because $0 \leq c \leq d(\boldsymbol{p}, D), \boldsymbol{p}_{c}:=\boldsymbol{e}\left(\frac{c}{d\left(\boldsymbol{p}, \boldsymbol{p}_{0}\right)}\right) \in \overline{\boldsymbol{p}_{0} \boldsymbol{p}}$. Then

$$
\left\{\boldsymbol{p}_{c}\right\}=\partial \mathcal{S}_{c}(D) \cap \overline{\boldsymbol{p}_{0} \boldsymbol{p}}
$$

because the offset surface $\partial \mathcal{S}_{c}(D)=\{\boldsymbol{x} \in X: d(\boldsymbol{x}, D)=c\}$ contains $\boldsymbol{p}_{c}$ since $d\left(\boldsymbol{p}_{c}, D\right)=c$; moreover, $[0,1] \ni t \rightarrow d(\boldsymbol{e}(t), D)$ function is strictly increasing, so the intersection is unique. This implies half of the proposed equality (5), because

$$
d\left(\boldsymbol{p}, \mathcal{S}_{c}(D)\right)=d\left(\boldsymbol{p}, \partial \mathcal{S}_{c}(D)\right) \leq d\left(\boldsymbol{p}, \boldsymbol{p}_{c}\right)=d\left(\boldsymbol{p}, \boldsymbol{p}_{0}\right)-d\left(\boldsymbol{p}_{0}, \boldsymbol{p}_{c}\right)=d(\boldsymbol{p}, D)-c .
$$

For the other direction, let us assume indirectly that $d\left(\boldsymbol{p}, \mathcal{S}_{c}(D)\right)<d\left(\boldsymbol{p}, \boldsymbol{p}_{c}\right)$, so there exist an $\boldsymbol{y}_{c} \in \mathcal{S}_{c}(D)$ such that $d\left(\boldsymbol{p}, \boldsymbol{y}_{c}\right)<d\left(\boldsymbol{p}, \boldsymbol{p}_{c}\right)$ as it is shown on Figure $4 \mathrm{~b}$. Since $D$ is a closed set, $\boldsymbol{y}_{c}$ also has a closest point in $D$ that we denote $\boldsymbol{y}_{0} \in D$. Using the definition for the distance, the triangle inequality in $\boldsymbol{y}_{c} \boldsymbol{y}_{0} \boldsymbol{p}$, the indirect assumption, and that

$$
d\left(\boldsymbol{y}_{c}, D\right)=d\left(\boldsymbol{y}_{c}, \boldsymbol{y}_{0}\right) \leq c,
$$

we arrive at a contradiction:

$$
\begin{aligned}
d(\boldsymbol{p}, D) & \leq d\left(\boldsymbol{p}, \boldsymbol{y}_{0}\right) \leq d\left(\boldsymbol{p}, \boldsymbol{y}_{c}\right)+d\left(\boldsymbol{y}_{c}, \boldsymbol{y}_{0}\right) \\
& <d\left(\boldsymbol{p}, \boldsymbol{p}_{c}\right)+c=d\left(\boldsymbol{p}, \boldsymbol{p}_{0}\right)=d(\boldsymbol{p}, D) .
\end{aligned}
$$

Remark. i). Because equation (6) is generally false for $t \notin[0,1], \boldsymbol{p}$ must not be inside $\mathcal{S}_{c}(D)$. 
ii). Note that the proof does not require that the closest point $\boldsymbol{p}_{0}$ to be unique, any one of them will suffice.

iii). Consider the signed distance function form of this theorem, Corollary 2. Because of equation (6), if $f$ is differentiable at point $\boldsymbol{x} \in \overline{\boldsymbol{p}_{0}} \subseteq \mathbb{R}^{3}$, then $\nabla f(\boldsymbol{x})=\frac{\boldsymbol{p}-\boldsymbol{p}_{0}}{\left\|\boldsymbol{p}-\boldsymbol{p}_{0}\right\|_{2}}$.

We can now state the theorem on offsetting SDFs:

Corollary 2 (Offset of an SDF). If $f: \mathbb{R}^{3} \rightarrow \mathbb{R}$ is an $S D F$, then for any $0 \neq c \in \mathbb{R}$ offset, the function $f-c$ is an $S D F$ on the set $\left\{\frac{f}{c} \geq 1\right\}$.

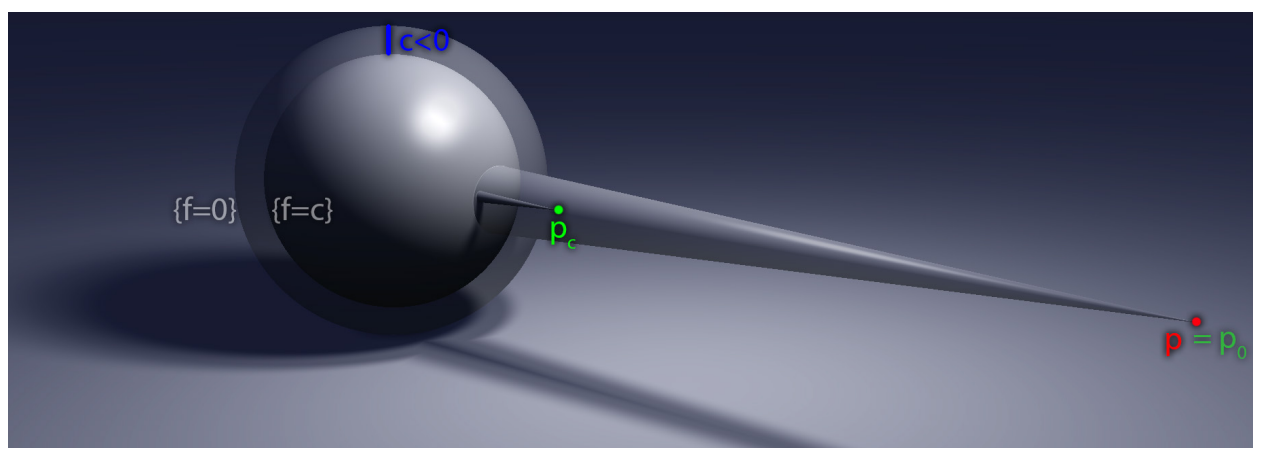

Figure 5: A counterexample for Corollary 2, when the condition does not hold.

Remark. i). $\left\{\frac{f}{c} \geq 1\right\}=\left\{\begin{array}{ll}\{f \leq c\} & \text { if } c<0 \\ \{f \geq c\} & \text { if } c>0\end{array}\right.$.

ii). The theorem is untrue for other points, as a counterexample is demonstrates this on Figure 5. Let $c<0$, and $\boldsymbol{p}$ be a point on a highly convex point on the surface as seen on the figure, so $\{f \equiv 0\} \ni \boldsymbol{p} \notin\left\{\frac{f}{c} \geq 0\right\}$. Then, let $\boldsymbol{p}_{0}$ be a closest point to $\boldsymbol{p}$ on the original surface $\{f \equiv 0\}$, and $\boldsymbol{p}_{c}$ be the closest point on offset surface $\{f \equiv c\}$. Clearly $\boldsymbol{p}=\boldsymbol{p}_{0}$, but because of the said convexity, $|c|<d\left(\boldsymbol{p}_{0}, \boldsymbol{p}_{c}\right)=d(\boldsymbol{p},\{f \equiv c\}) ;$ and therefore, $d(\boldsymbol{p},\{f \equiv 0\})-c=-c \neq$ $d(\boldsymbol{p},\{f \equiv c\})$.

\section{Conclusion}

This paper presented a theoretical overview of surfaces defined by signed distance functions. We formulated equivalent definitions to emphasize the geometric properties of this implicit representation.

We defined an abstract offset set of an arbitrary set in Banach spaces. Our main theoretical contribution is a theorem stating a distance equivalence for points outside of the offset set. 
Most importantly, Theorem 1 exposes a way to compute a signed distance function of an offset surface defined by an SDF by merely subtracting the offset radius from the function. However, this formulation is limited to the exterior of the offset volume, and the error can be arbitrarily large as we demonstrated on Figure 5.

The simple subtraction formula for offsetting a signed distance function was often used in practice, but it was only validated empirically. Our paper gave this missing guarantee and explained when this formula does not work.

\section{References}

[1] Aaltonen, Sebastian. GPU-based clay simulation and ray-tracing tech in Claybook. San Francisco, CA, March 2018. Game Developers Conference.

[2] Angles, Baptiste, Tarini, Marco, Wyvill, Brian, Barthe, Loïc, and Tagliasacchi, Andrea. Sketch-based implicit blending. ACM Trans. Graph., 36(6):181:1181:13, November 2017. DOI: 10.1145/3130800.3130825.

[3] Bernhardt, Adrien, Barthe, Loic, Cani, Marie-Paule, and Wyvill, Brian. Implicit blending revisited. In Computer Graphics Forum, volume 29, pages 367-375. Wiley Online Library, 2010.

[4] Bloomenthal, Jules and Wyvill, Brian, editors. Introduction to Implicit Surfaces. Morgan Kaufmann Publishers Inc., San Francisco, CA, USA, 1997.

[5] Dyer, R.H. and Edmunds, D.E. From Real to Complex Analysis. Springer Undergraduate Mathematics Series. Springer International Publishing, 2014.

[6] Evans, Alex. Learning from failure: a survey of promising, unconventional and mostly abandoned renderers for 'dreams ps4', a geometrically dense, painterly ugc game. In Advances in Real-Time Rendering in Games. MediaMolecule, SIGGRAPH, 2015.

[7] Gomes, Abel, Voiculescu, Irina, Jorge, Joaquim, Wyvill, Brian, and Galbraith, Callum. Implicit Curves and Surfaces: Mathematics, Data Structures and Algorithms. Springer Publishing Company, Incorporated, 1st edition, 2009.

[8] Gourmel, Olivier, Pajot, Anthony, Paulin, Mathias, Barthe, Loic, and Poulin, Pierre. Fitted BVH for Fast Raytracing of Metaballs. Computer Graphics Forum, 2010. DOI: 10.1111/j.1467-8659.2009.01597.x.

[9] Hansen, C., Hijazi, Y., Hagen, H., Knoll, A., and Wald, I. Interactive ray tracing of arbitrary implicits with simd interval arithmetic. In IEEE/ EG Symposium on Interactive Ray Tracing 2007(RT), volume 00, pages 11-18, 09 2007. DOI: 10.1109/RT.2007.4342585.

[10] Hart, John C. Sphere tracing: A geometric method for the antialiased ray tracing of implicit surfaces. The Visual Computer, 12:527-545, 1994. 
[11] Hutchinson, John E. Introduction to Mathematical Analysis. Australian National University Lecture Notes, 1994.

[12] Malladi, Ravikanth, Sethian, James A., and Vemuri, Baba C. Shape modeling with front propagation: A level set approach. IEEE Trans. Pattern Anal. Mach. Intell., 17(2):158-175, February 1995. DOI: 10.1109/34.368173.

[13] Pasko, A., Adzhiev, V., Sourin, A., and Savchenko, V. Function representation in geometric modeling: concepts, implementation and applications. The Visual Computer, 11(8):429-446, Aug 1995. DOI: 10.1007/BF02464333.

[14] Ricci, A. A Constructive Geometry for Computer Graphics. The Computer Journal, 16(2):157-160, May 1973. DOI: http://dx.doi.org/10.1093/comjnl/16.2.157.

[15] Shapiro, Vadim. Semi-analytic geometry with r-functions. Acta Numerica, 16:239-303, 2007. DOI: 10.1017/S096249290631001X.

[16] Sherstyuk, Andrei. Fast ray tracing of implicit surfaces. Computer Graphics Forum, 18(2):139-147. DOI: 10.1111/1467-8659.00364.

[17] Szécsi, László and Illés, Dávid. Real-time metaball ray casting with fragment lists. In Andújar, Carlos and Puppo, Enrico, editors, Eurographics (Short Papers), pages 93-96. Eurographics Association, 2012.

[18] Wright, Daniel. Dynamic occlusion with signed distance fields. In Advances in Real-Time Rendering in Games. Epic Games (Unreal Engine), SIGGRAPH, 2015.

[19] Wyvill, Brian, Guy, Andrew, and Galin, Eric. Extending the CSG Tree. Warping, Blending and Boolean Operations in an Implicit Surface Modeling System. Computer Graphics Forum, 1999. DOI: 10.1111/1467-8659.00365. 